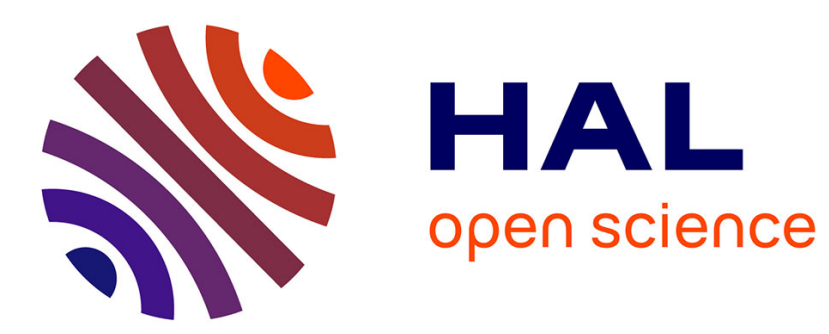

\title{
Spice-Compatible High Frequency Physical Modeling Approach for an Inductor
}

\author{
Jad Taki, Mohamed Bensetti, Daniel Sadarnac
}

\section{To cite this version:}

Jad Taki, Mohamed Bensetti, Daniel Sadarnac. Spice-Compatible High Frequency Physical Modeling Approach for an Inductor. 2015 9th International Conference on Power Electronics and ECCE Asia (ICPE-ECCE Asia), Jun 2015, Seoul, South Korea. 10.1109/ICPE.2015.7167783 . hal-01215832

\section{HAL Id: hal-01215832}

https://hal-centralesupelec.archives-ouvertes.fr/hal-01215832

Submitted on 15 Oct 2015

HAL is a multi-disciplinary open access archive for the deposit and dissemination of scientific research documents, whether they are published or not. The documents may come from teaching and research institutions in France or abroad, or from public or private research centers.
L'archive ouverte pluridisciplinaire HAL, est destinée au dépôt et à la diffusion de documents scientifiques de niveau recherche, publiés ou non, émanant des établissements d'enseignement et de recherche français ou étrangers, des laboratoires publics ou privés. 


\title{
SPICE-Compatible High Frequency Physical Modeling Approach for an Inductor
}

\author{
J. TAKI ${ }^{1}$, M. BENSETTI ${ }^{1}$, and D. SADARNAC ${ }^{1}$ \\ ${ }^{1}$ GeePs | Group of electrical engineering - Paris \\ UMR CNRS 8507, CentraleSupelec, Univ Paris-Sud, Sorbonne Universités, UPMC Univ Paris 06 \\ 11, rue Joliot-Curie, Plateau de Moulon, F91192 Gif-sur-Yvette, France
}

\begin{abstract}
Multi-physics modeling in power electronics contains EMC studies, thus conception requires modeling of passive elements. These models need to be compatible with the simulation software usually used in the industry. An analysis for modeling an inductor at high-frequency is presented in this study. The method takes into account the proper inductances of the turns, the mutual inductances between them, the parasitic capacitances, and the resistance of the windings. The originality of the model is that it is based on physical geometries and the material characteristics of the inductor and covers a large frequency bandwidth. These elements are used to create an analytical model based on electrical equations in order to provide the frequency dependent impedance of the inductor. The model is validated using experimental measurements.
\end{abstract}

Index Terms- EMC, High Frequency, Physical Modeling of Inductor, SPICE.

\section{INTRODUCTION}

Multi-physics modeling in power electronics contains EMC studies, thus conception requires modeling of passive elements. These models need to be physical in order to be more effective, compatible with the simulation software usually used in the industry, and predictive (before the prototyping phase).

At high frequencies, these models will have a different behavior than at low frequencies. Many factors can affect this behavior and force the components of any model to be frequency-dependent.

Previous studies have used a number of different methods to model high frequency inductors. Some are based on the finite element method (FEM)[10], which consumes a lot of hardware and time, and can only provide a global model that does not take into consideration the parasitic elements of the inductor.

Other models are physical analytical models based on lumped parameters that give an equivalent circuit model with global frequency-dependent $\mathrm{L}, \mathrm{C}$ and $\mathrm{R}$ $[1][2][7][8][11]$. These models are not optimized for use in an electric circuit simulator such as SPICE. [12]

A number of methods represent the non-linear core model as a series of LR branches in order to take into consideration the hysteresis cycle [3]. These models do not account for the physical parasitic seen at high frequencies and also replace the capacitances between coils by one equivalent capacitor. Such modifications can influence the time domain simulations as the model does not completely reproduce reality.
The purpose of this study is to give a physical model of an inductor based on its geometry and the characteristic of its core and windings while taking into account the inductances, the capacitances, and the core losses which exist. These parameters are defined using formulas from the literature [2][4][5][6][9].The model will represent the real aspect and behavior of the inductor at both low and high frequencies, being a physical model rather than a lumped parameter equivalent model.

The study models each turn by the electrical equation between 2 nodes. Following this, all capacitances between nodes are included in order to define a resonant model of the inductor. The frequency response of this model is compared to a real measurement in order to validate it. Finally, a time domain simulation of the model is presented.

\section{Model Definition}

\section{A. Structure}

The inductor consists of $\mathrm{n}$ turns on the same layer as shown in fig. 1. The approach involves modeling each turn based on its physical and electromagnetic characteristics as well as modeling the coupling between the turns.

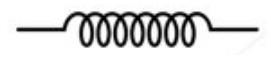

Fig. 1: Inductor of $\mathrm{n}$ turns on the same layers

Fig.2 shows an electromagnetic representation of a winding where $R_{i}$ represents the resistance of the turn $\mathrm{i}$ (including the core losses), $L_{i}$ is its proper inductance , and $M_{i j}$ is the mutual inductance between turns $i$ and $j$.

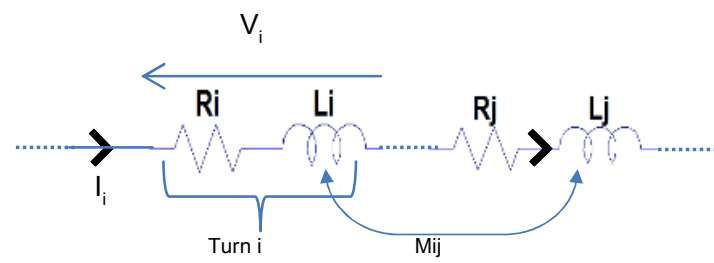

Fig. 2: Electromagnetic Model of a winding

As for the electrostatic system, the parasitic capacitances between the turns (consecutive or not) need 
to be integrated in the system. The next subsections will present the method of calculation used for determining the parameters mentioned above.

\section{B. Proper and Mutual Inductances}

Grover [6] presented formulas and methods for calculating the proper and mutual inductances for different coil forms.

This work focusses on coaxial circular turns. The proper inductance for turn $i$ can be calculated using (1):

$$
L_{i}=\mu R\left[\ln \left(\frac{8 R}{r}\right)-\frac{7}{4}\right]
$$

where $\mu$ is the permeability of the core, $r$ the radius of the conductor, and $\mathrm{R}$ the radius of the turn.

For the mutual inductance calculation, we consider having two turns $i$ and $j$ as shown in fig. 3. Based on Neumann's formula, the mutual inductance between the two turns in question is determined as follows:

$$
M_{i j}=\frac{\mu}{4 \pi} \iint \frac{\overrightarrow{d s_{l}} \cdot \overrightarrow{d s_{J}}}{d}
$$

where $\mathrm{ds}_{\mathrm{i}}$ and $\mathrm{ds}_{\mathrm{j}}$ are incremental sections of the conductors and $\mathrm{d}$ is the distance between them.

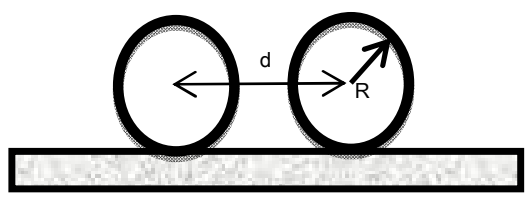

Fig. 3: Cross-section of 2 turns

The integral in (2) can be represented in terms of the complete elliptic functions $\mathrm{K}$ and $\mathrm{E}$ in (3) and (4):

$$
\begin{gathered}
K(k)=\int_{0}^{\frac{\pi}{2}} \frac{d \varphi}{\sqrt{1-k^{2}(\sin \varphi)^{2}}} \\
E(k)=\int_{0}^{\frac{\pi}{2}} \sqrt{1-k^{2}(\sin \varphi)^{2}} d \varphi
\end{gathered}
$$

Using these elliptic functions, the mutual inductance between turn $i$ and turn $j$ is determined as in (5):

$$
M_{i j}=2 \mu \frac{\sqrt{a+b}}{b}\left[\left(a-\frac{\beta^{2}}{2}\right) K(\beta)-E(\beta)\right]
$$

where:

$$
a=\frac{2 R^{2}+d^{2}}{4 R^{2}}
$$

$$
\begin{gathered}
b=\frac{2}{R^{2}} \\
\beta=\sqrt{\frac{2 b}{a+b}}
\end{gathered}
$$

Considering a solenoid consisting of $\mathrm{n}$ turns, for each, it is necessary to determine the proper inductance and mutual inductance between the turn in question and all other turns. Knowing all inductances in the structure, we the total inductance of the solenoid is obtained:

$$
L_{t o t}=\sum_{i=1}^{n} L_{i}+\sum_{j=1}^{n-1} 2(n-j) M_{1(j+1)}
$$

This factor gives a good image of the inductor in the first frequency range (before the main resonance frequency) where the parasitic parameters of the inductor will be neglected beside the inductive dominance.

(1) and (2) show that the proper and mutual inductances depend on the permeability of the core. In the case of a solenoid (rod), the electromagnetic field runs outside the core and closes its circuit through the surrounding air. This will change the effective permeability of the core. Instead of having:

$$
\mu=\mu_{r} \mu_{0}
$$

where $\mu_{\mathrm{r}}$ is the relative permeability of the core and $\mu_{0}$ the permeability of air, the relative permeability will be replaced by an effective permeability. This last depend on the core geometry as well as its relative permeability and can be obtained using the plot in fig. 4 [13]

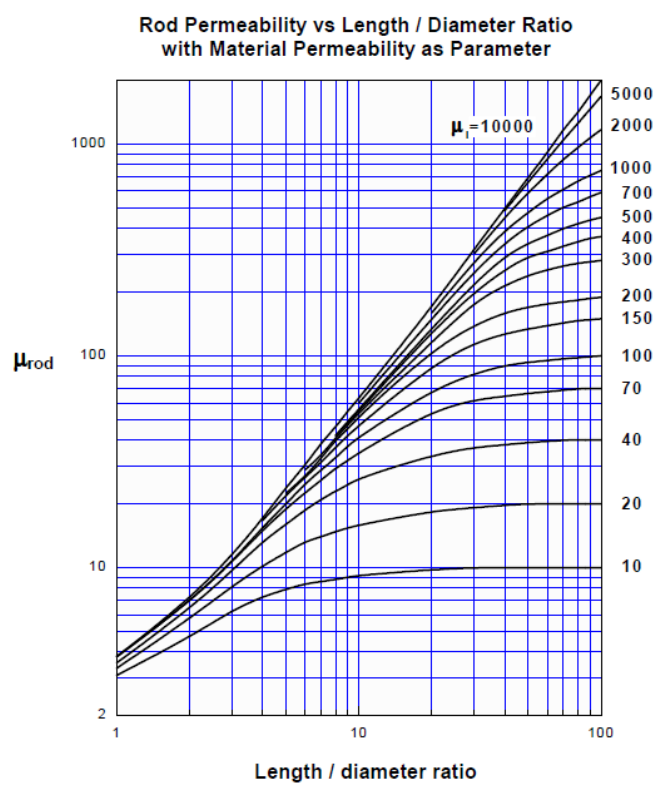

Fig. 4: Effective permeability of rods 


\section{Capacitances}

In this section, the calculation of the capacitances between turns or between a turn and the core will be presented. The impedance of the capacitance is inversely proportional to the frequency. At low frequencies, this impedance will be neglected compared to the inductance's which is directly proportional to the frequency. In order to model an inductor at high frequency, it is important to determine the value of these capacitances and to integrate them in the model.

In [4], A. Massarini proposed a method to determine these capacitances by studying the lines of the electric field. Considering 2 conductors as shown in fig. 5, the electric field between the 2 conductors is present in the insulating coating as well as in the air between the 2 conductors.

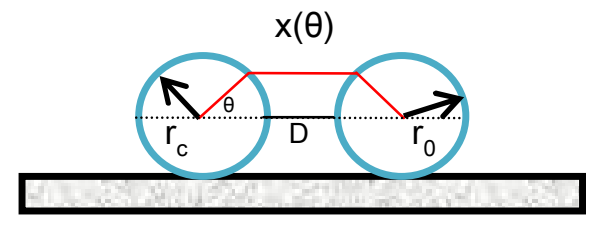

Fig. 5: Cross Section of 2 conductors with the

The elementary capacitance of the insulating coatings can be determined as mentioned in (8):

$$
d C_{t t c}=\frac{\varepsilon_{r} \varepsilon_{0} l_{t}}{2 * \ln \frac{r_{0}}{r_{c}}} d \theta
$$

where $l_{t}$ is the turn length, $r_{c}$ the inner radius and $r_{0}$ the outer radius.

As for the capacitance of the air gap, it is necessary to determine the path of the electric field:

$$
x(\theta)=2 R_{0}(1-\cos \theta)+D
$$

The elementary surface of the wire is:

$$
d S=\frac{l_{t} * r_{0}}{2}
$$

Using (9) and (10), we can determine the elementary capacitance per unit angle of the air gap:

$$
\frac{d C_{g}(\theta)}{d \theta}=\varepsilon_{0} \frac{d S}{x(\theta)}
$$

The series combination of the elementary capacitances mentioned above is given by:

$$
d C_{e q}(\theta)=\frac{d C_{t t c} d C_{g}}{d C_{t t c}+2 d C_{g}}
$$

And finally, to obtain the capacitance between the turns, (12) is integrated:

$$
C_{t t}=2 \int_{0}^{\pi / 6} d C_{e q}
$$

The equation above can be used to calculate the turnto-core capacitance. Since the core is at a constantpotential and located on the vertical plane of the conductors as shown in Fig. 2, the path lengths of the electric field studied above in the air gap between the turn and the core are one half of the path lengths between two conductors on the same layer. As a result, the capacitance between a turn and the core can be determined:

$$
C_{t c}=2 C_{t t}
$$

The formulas presented in this subsection permit the determination of every parasitic capacitance in our system whether it was an adjacent turn-to-turn capacitance, non-adjacent turn-to-turn capacitance, or turn-to-core capacitance.

\section{Resistances}

The system resistance is due to both the winding resistances $R_{w}$ and the core series resistance $R_{c}$. Both of these resistances are frequency-dependent. For the winding resistance, skin-effect and proximity-effect should be taken into consideration. An analytical representation of these effects is shown in (15) [1]:

$$
R_{w}=R_{w d c} A\left[\frac{e^{2 A}-e^{-2 A}+2 \sin (2 A)}{e^{2 A}+e^{-2 A}-2 \cos (2 A)}\right]
$$

where $R_{w d c}$ is the DC resistance and A for a round wire is:

$$
A=\left(\frac{\pi}{4}\right)^{0.75} \frac{d^{1.5}}{\delta t^{0.5}}
$$

In (16), $\mathrm{d}$ represents the conductor diameter and $\mathrm{t}$ the distance between the centers of two adjacent conductors. The skin depth of the conductor $\delta$ is given by:

$$
\delta=\sqrt{\frac{\rho_{w}}{\pi \mu_{0} f}}
$$

where $\rho_{w}$ is the wire resistivity.

On the other hand, the frequency-dependent core resistance needs the knowledge of the frequencydependent permittivity and permeability of the core.

The frequency-dependent permeability represents the core magnetic hysteresis loss:

$$
\hat{\mu}_{c}=\mu_{0} \hat{\mu}_{r c}=\mu_{0}\left(\mu_{r c}^{\prime}-j \mu_{r c}^{\prime \prime}\right)
$$


To include the core dielectric loss, the electric permittivity of the core is presented by a complex quantity that is also frequency-dependent:

$$
\hat{\varepsilon}_{c}=\varepsilon_{0} \hat{\varepsilon}_{r c}=\varepsilon_{0}\left(\varepsilon_{r c}^{\prime}-j \varepsilon_{r c}^{\prime \prime}\right)
$$

Using these definitions, the core series resistance can be calculated as shown in (20):

$$
R_{c}=-\frac{4 \pi}{\mu_{r c}^{\prime}} f L_{d c} \operatorname{Im}\left[\frac{\hat{\mu}_{r c}}{j^{1.5} \sqrt{2} \frac{r_{0}}{\widehat{\delta}}} \frac{J_{1}\left(j^{1.5} \sqrt{2} \frac{r_{0}}{\hat{\delta}}\right)}{J_{0}\left(j^{1.5} \sqrt{2} \frac{r_{0}}{\hat{\delta}}\right)}\right]
$$

The resistance in (20) is represented using the Bessel function of first kind,

$$
J_{n}(x)=\frac{1}{2 \pi} \int_{-\pi}^{\pi} e^{i(n \tau-x \sin (\tau))} d \tau
$$

and $\delta$ :

$$
\delta=\sqrt{\frac{1}{\pi f \hat{\mu}_{c}\left(\delta_{c}+j 2 \pi f \hat{\varepsilon}_{c}\right)}}
$$

where $\delta_{c}$ is the electric conductivity of the core.

In this section, the determination of the frequencydependent resistance was presented, which is the series association of the conductor resistance and the core equivalent resistance.

\section{E. SPICE Model}

The purpose of this work is to develop a Spice compatible physical model. In order to do that, every section studied must be represented using Spice models. To model a solenoid with $n$ turns in Spice, the system is considered to be composed at first of $n+1$ major nodes in series. Each turn will be represented between two nodes.

The electromagnetic system will be modeled by arbitrary behavioral voltage sources between two major nodes. The proper and mutual inductances in the system can be calculated as mentioned in section II-B. Using (23) for each turn $i$ models the entire electromagnetic system of the solenoid:

$$
V_{i}=L_{i} \frac{d I_{i}}{d t}+\sum_{\substack{j=1 \\ j \neq i}}^{n} M_{i j} \frac{d I_{j}}{d t}
$$

where $I_{i}$ is the current in the turn $i$.

In addition to the arbitrary behavioral voltage, the wire and core resistance calculated in section II-D were added in series. Fig 6 represents the model for turn $i$ which includes each of these elements:

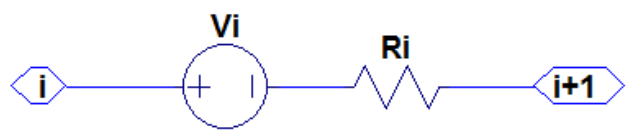

Fig. 6: Modeling a turn of the solenoid in Spice

After modeling all turns in such a way, it is necessary to add the electrostatic system. In section II-C, it was shown how to determine the capacitance between two turns on the same layer or between a turn and the core. The capacitance between the turn $i$ and $j$ will be placed between the node $i$ and the node $j$. On the other hand, the capacitance between the turn $i$ and the core will be placed between the node $i$ and a common node for all the turns $N_{\text {core }}$ Fig. 7 shows a portion of the model with the capacitances added.

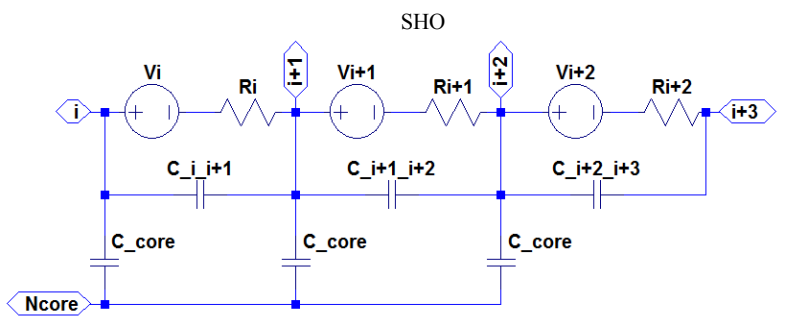

Fig. 7: A part of the model showing the 3 parts studied

\section{EXPERIMENTAL VALIDATION}

In order to validate our model, experimental results are necessary. Fig. 8 shows a solenoid prepared in our laboratory in order to be used in this study:

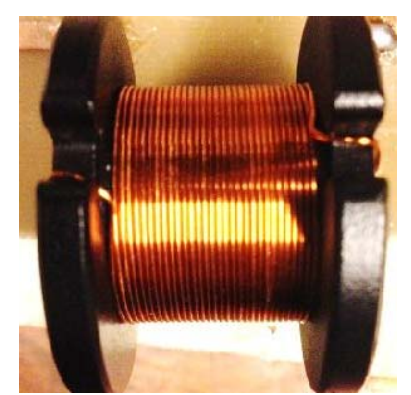

Fig. 8: Solenoid used to validate the model

The solenoid in question is composed of a single layer over a ferrite bobbin core. The geometric characteristics are listed as follows:

- $\mathrm{R}$ : Radius of the solenoid: $15 \mathrm{~mm}$

- $\mathrm{r}$ : Radius of the conductor: $0.25 \mathrm{~mm}$

- e : Distance between 2 adjacent turns : 0.54 $\mathrm{mm}$

- $\mathrm{n}:$ Number of turns : 31

- $\mathrm{u}_{\mathrm{reff}}$ : Effective relative permeability of the core: 4.3

In order to measure the impedance of the solenoid, an Agilent E5071C Network Analyzer has been used (fig. 9). 


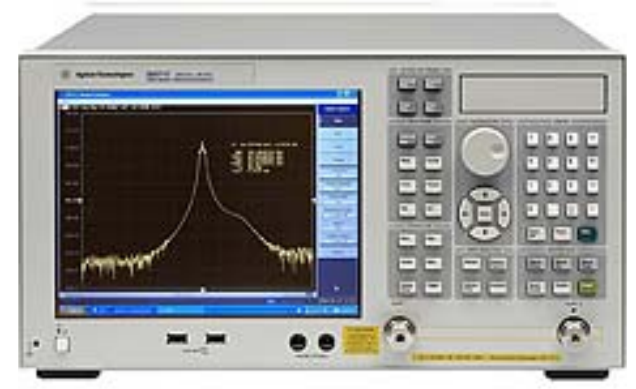

Fig. 9: Agilent E5071C Network Analyzer

The device will measure the $S_{i j}$ parameters as a function of frequency. These parameters will be used in order to calculate the impedance of the solenoid and thus its real part, imaginary part, module and phase.

Fig. 10 and Fig. 11 shows a comparison between the real part of the real solenoid and the physical model proposed in this article. It can be seen that the real part of the impedance of the model is close to real measurements. For low frequencies, the real part does not extend few Ohms and as soon as we approach the first resonance, it increases exponentially and hits its maximum value as the mentioned frequency. From there, increasing the frequency results in decreasing the impedance real part exponentially.

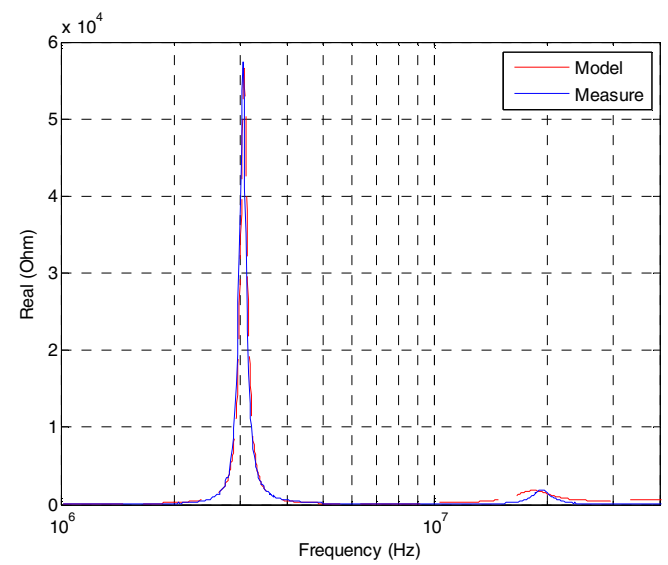

Fig. 10: Real part of the solenoid

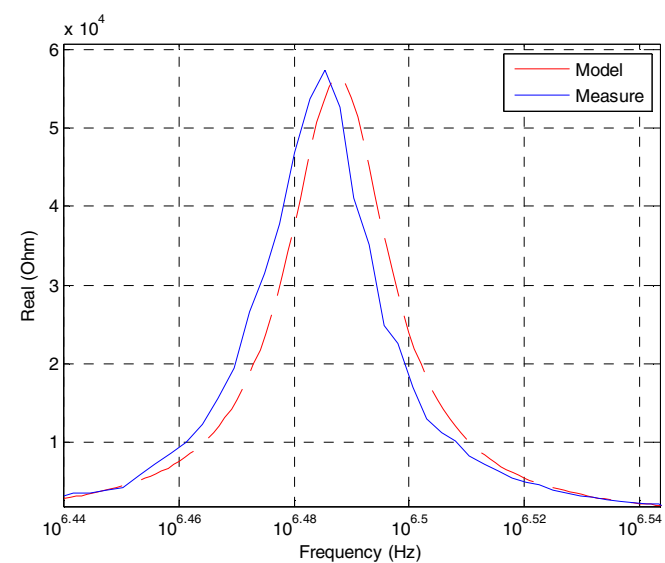

Fig. 11: Real part of the solenoid (with Zoom)

For the imaginary part, a comparison between the model and the measurement is shown in Fig. 12. Before the first resonant frequency, the imaginary part is positive as the solenoid has an inductance aspect. Resonance is visible when the imaginary part is null which first occurs at a frequency of $3.07 \mathrm{MHz}$. The negative part reflects the capacitive aspect of the solenoid. Fig. 12 also shows the validity of the model, comparing well to the measurements.

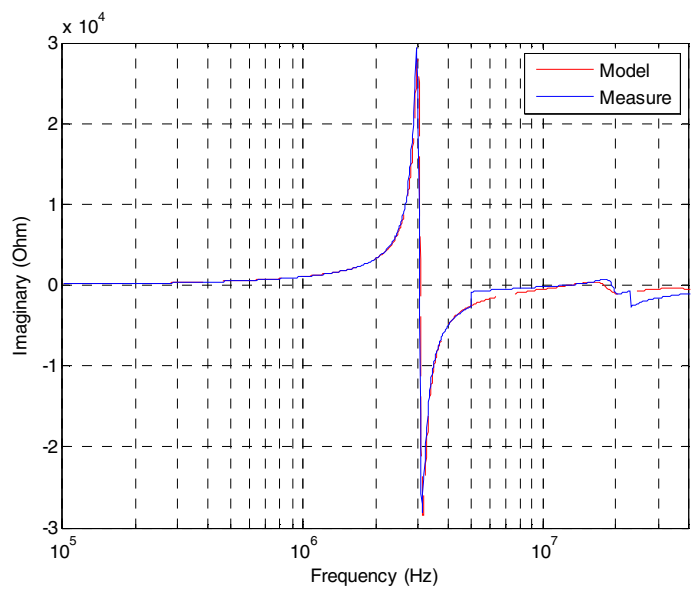

Fig. 12: Imaginary part of the solenoid

Fig. 13 compares the module of the impedance of the model presented in this work and the measurements.

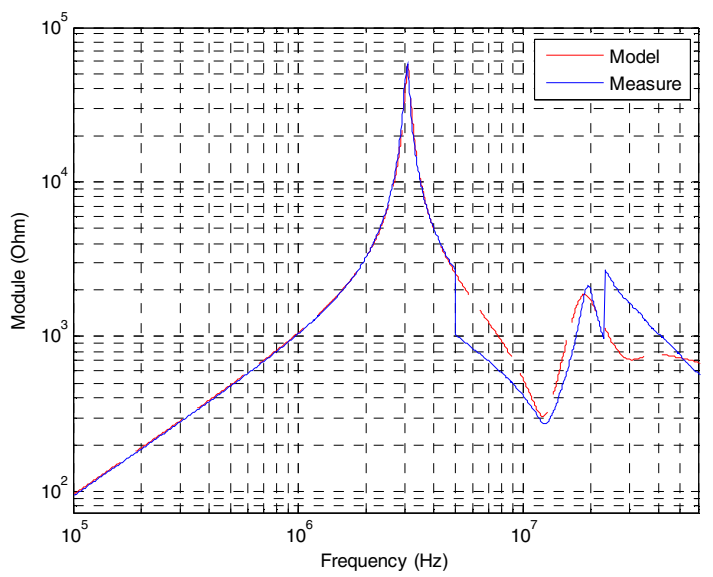

Fig. 13: Impedance Module of the solenoid

It can be seen that the comparison between the proposed model and the measurements shows a good agreement until a certain frequency. Below the first resonant frequency, there is little difference between the model and the measurements. When the frequency reaches $5 \mathrm{MHz}$, slight differences can be seen but the model continue is still able to locate a second and third resonance at $12 \mathrm{MHz}$ and $20 \mathrm{MHz}$. Above these frequencies, the limitations of the model prevent comparison. 
As previously mentioned, the model proposed in this study can used in both time and frequency domains. In the previous section, the frequency-domain validation has been studied. The efficiency of the model in the time domain must also be verified. In order to do this, the proposed model is excited by a current source of $1 \mathrm{~A}$ and the voltage at its terminals is studied. Fig. 14 presents the result of the time simulation.

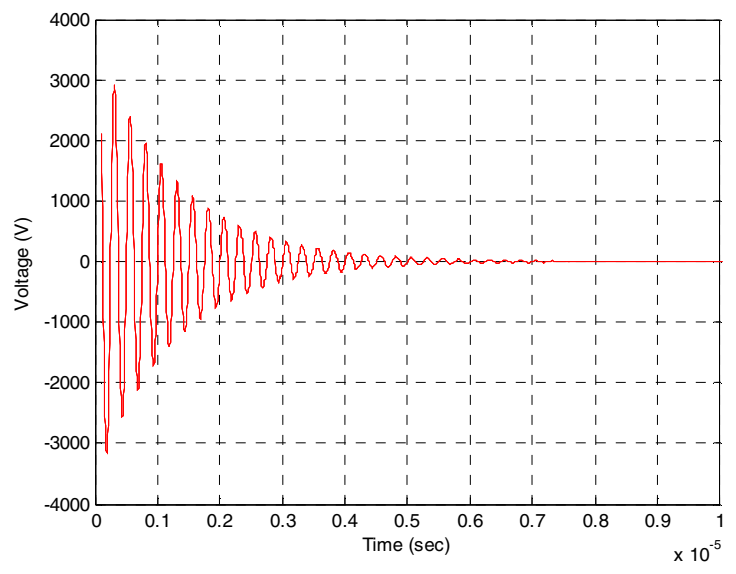

Figure 14: Time simulation of the model

In this figure, two regions can be found in the simulation. The transient state shows the effect of the parasitic elements in the solenoid and is presented by a harmonic oscillator. The inductances and capacitances in the system have an effect on the frequencies of the oscillations. On the other hand, the resistances present in the model will change the oscillations amplitude. As for the permanent state, it's a constant voltage relative to the resistive part of the inductor.

The last simulation shows the response of the inductor model to a constant current. In power electronics, currents switch quickly in the presence of active components. Fig. 15 shows the response of our model to a pulse signal.

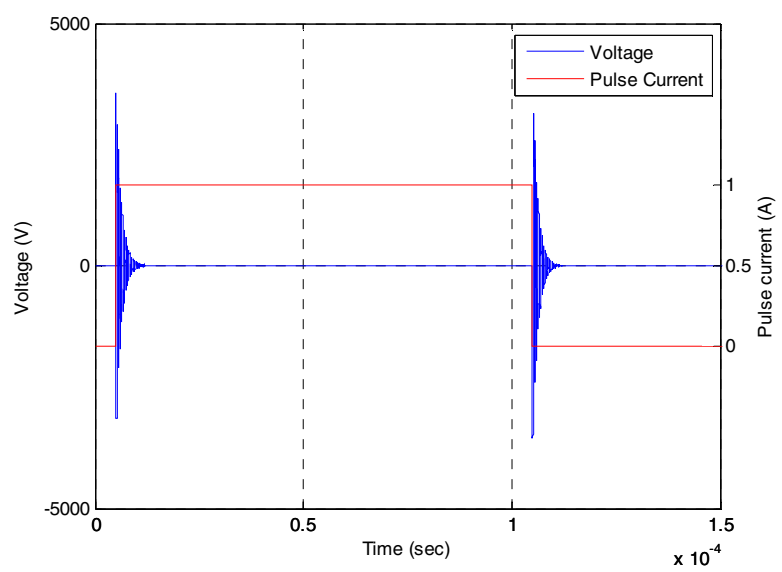

Figure 15: Voltage response of the model to a pulse current
An approach providing a physical model of an inductor based on its geometry and magnetics and electrostatic characterization has been introduced here. This model gives a better understanding of the behavior at high frequencies and parasites and is SPICE compatible. Previous models which can be used in SPICE did not give this level of precision at high frequencies. This work provides an efficient model both in the frequency (low and high frequencies) and the time domains.

\section{REFERENCES}

[1] M.K. Kazimierczuk and G. Grandi, "High-Frequency SmallSignal Model of Ferrite Core Inductors," IEEE Transactions on Magnetics, vol. 35, no. 5, pp. 4185-4191, September 1999.

[2] A. Massarini, M.K. Kazimierczuk and G. Grandi, "Lumped Parameter Models for Single and Multiple-Layer Inductors," in IEEE 1996 Power Electronics Specialists Conference, 1996, pp. 295-301 vol 1.

[3] L. Heinemann, R. Schulze, P. Wallmeier and H. Grotsollen, "Modeling of High Frequency Inductors," in IEEE 1994 Power Electronics Specialists Conference, 1994, pp. 876-883 vol 2.

[4] A. Massarini and M.K. Kazimierczuk, "Self-Capacitances of Inductors," IEEE Transactions on Power Electronics, vol. 12, no. 4, pp. 671-676, 1999.

[5] G. Grandi, M.K. Kazimierczuk, A. Massarini and U. Reggiani, "Stray Capacitances of Single-Layer Solenoid Air-Core Inductors," IEEE Transactions on Industry Applications, vol. 35, no. 5, pp. 1162-1168, 1999.

[6] Grover, F. W., Inductance Calculations, Chaps. 2 and 13, Dover, New York, 1964.

[7] D. LIU and J. JlANG, "High frequency model of common mode inductor for EM1 analysis based on measurements," in IEEE 2002 3rd International Symposium on Electromagnetic Compatibility, 2002, pp. 462-465 vol 1.

[8] G. Grandi, M.K. Kazimierczuk, A. Massarini and U. Reggiani, "Model of Laminated Iron-Core Inductors for High Frequencies," IEEE Transactions on Magnetics, vol. 40, no. 4, pp. 1839-1845, 2004.

[9] M.J. Hole and L.C. Appel, "Stray capacitance of a twolayer air-cored inductor," IEE Proc.-Circuits Devices Syst., Vol. 152, No. 6, pp. 565-572, December 2005

[10] A. ZHOU, "Modèles de composants passifs et couplage électromagnétique pour filtres HF de puissance - Optimisation du placement," PhD thesis of Lyon University, 2012

[11] A. Ragusa, P. Zanchetta, L. Empringham, L. De Lillo, M. Degano - "High Frequency Modelling method of EMI filters for Hybrid $\mathrm{Si}$ - SiC Matrix Converters in Aerospace Applications", Applied Power Electronics Conference and Exposition (APEC), 2013 Twenty-Eighth Annual IEEE, pp. $2610-2617$.

[12] http://www.linear.com/

[13] http://www.magneticsgroup.com/pdf/erods.pdf 